\title{
Rapid Eye Movement Sleep: Regulation and Function
}

\author{
Birendra N. Mallick, S.R. Pandi-Perumal, Robert W. McCarley, and Adrian R. Morrison, editors; \\ Cambridge University Press, New York, NM 2011; \$165; ISBN: 978-0-521-11680-0 \\ Frank M. Ralls, M.D. \\ Division of Pulmonary, Critical Care, and Sleep Medicine, Department of Internal Medicine and the University of New Mexico \\ Hospital Sleep Disorders Center, Albuquerque, NM
}

$R$ apid Eye Movement Sleep: Regulation and Function is an expertly crafted treatise on rapid eye movement (REM) sleep, aimed at the reader with particular interest in this phenomenon. The discovery of REM sleep serves as an important lesson in the role of "thinking outside of the box" in the advance of science. Prior to 1951, the prevailing thought in academic circles was that sleep was a passive phenomenon. Our knowledge of sleep physiology took a quantum leap forward in that year when University of Chicago graduate student Eugene Aserinsky, using an aging Offner Dynograph, enlisted his 8-year-old son, Armond, for a recording of the youngster's electroencephalogram and electrooculogram during sleep. Later in the night, the recording appeared to show that his son was awake and looking around, prompting Aserinsky to check on Armond's well-being. Rather than awake, on the contrary he found Armond with his eyes closed and clearly asleep. Aserinsky and his mentor Nathaniel Kleitman went on to publish a brief report of this intriguing finding in the journal Science, and the field of rapid eye movement sleep was born. Since this initial discovery, the images, ideas, sensations, and emotions associated with REM sleep have been the topic of philosophical, religious, and scientific speculation, and a burgeoning amount of research has appeared aimed at identifying and defining the unique characteristics of this physiologically different phase of sleep. Rapid Eye Movement Sleep: Regulation and Function serves as a compilation of both the extant body of scientific research on REM sleep (and dreaming), as well as touching on some of the psychological and philosophical implications of this state of being.

This is a weighty volume of 478 pages, divided into six provocative sections. The introductory chapters present a history of the science of dreaming and REM sleep, a discussion of the inexact temporal association between REM sleep and dreaming, and philosophical musings on the phenomenology of dreaming. Chapters on the hard science of REM follow, with extensive discussions of general biology in section II (including evolutionary development, a systems approach to REM sleep, and circadian considerations), and then chapters in sections III, IV, and V exploring topics such as the interactions between various neuroanatomical locations that regulate and control REM, the neurochemistry of REM, and the functional significance of REM. There are detailed descriptions of the research that has led to our current understanding of the neuroanatomy and neurochemistry of REM; the interested reader will be fascinated by the descriptions of these painstaking investigations. The book also describes certain specific CNS locations that are activated by REM sleep, presumably leading to the experiencing of emotions or memories during this sleep state. After extensive coverage of normal REM sleep, section VI concludes the text by discussing what can go wrong in REM sleep, particularly narcolepsy, REM sleep behavior disorder (RBD), and multi-directional interactions between psychiatric illness, REM sleep, and dreaming. Although the title of the book implies that the focus is on REM sleep alone, many subjects that are important to the regulation of NREM sleep and of wakefulness appear throughout the text as well. Particularly noteworthy are concise discussions of the neurotransmitters that are essential to the regulation of wake, NREM sleep, and REM sleep. Accompanying all of this material are exceptional colored pictures, graphs, MRI scans, slides, and other excellent visual learning aids.

Rapid Eye Movement Sleep: Regulation and Function condenses nearly 60 years of research and academic thought into a wellwritten and nicely illustrated review of virtually every aspect of this intriguing state of being. Although there are books that speak to the subjects of sleep and dreaming, most are not scientifically rigorous. This book is unique in view of its meticulous, scientifically precise focus. While this approach may appear daunting at first, the body of knowledge presented is in fact easily accessible and consequently presents an opportunity for the reader to be exceptionally well versed in a fascinating aspect of sleep medicine. Definitely an excellent addition to the sleep specialist's library.

\section{CITATION}

Ralls FM. Rapid eye movement sleep: regulation and function. J Clin Sleep Med 2013;9(6):635.

\section{SUBMISSION \& CORRESPONDENCE INFORMATION}

Submitted for publication January, 2013

Accepted for publication January, 2013

Address correspondence to: Frank M. Ralls, M.D., Assistant Professor of Internal Medicine, Medical Director, Adult Sleep Medicine Services and Program Director, Fellowship in Sleep Medicine, University of New Mexico Hospital Sleep Disorders Center, 1101 Medical Arts Avenue NE, Building \#2, Albuquerque, NM 87102; Tel: (505) 272-6110; Fax: (505) 272-6112; E-mail: fralls@salud.unm.edu

\section{DISCLOSURE STATEMENT}

The author has indicated no financial conflicts of interest. 